\title{
Effect of Time Interval between Breast-Conserving Surgery and Radiation Therapy on Outcomes of Node-Positive Breast Cancer Patients Treated with Adjuvant Doxorubicin/Cyclophosphamide Followed by Taxane
}

\author{
Hyeon Kang Koh, $\mathrm{MD}^{1,2}$ \\ Kyung Hwan Shin, MD ${ }^{1,3}$ \\ Kyubo Kim, MD' \\ Eun Sook Lee, MD ${ }^{3}$ \\ In Hae Park, MD \\ Keun Seok Lee, MD ${ }^{3}$ \\ Jungsil Ro, MD \\ So-Youn Jung, $\mathrm{MD}^{3}$ \\ Seeyoun Lee, MD ${ }^{3}$ \\ Seok Won Kim, MD ${ }^{3}$ \\ Han-Sung Kang, MD \\ Eui Kyu Chie, MD \\ Wonshik Han, MD ${ }^{4}$ \\ Dong-Young Noh, $\mathrm{MD}^{4}$ \\ Kyung-Hun Lee, $\mathrm{MD}^{5}$ \\ Seock-Ah Im, MD ${ }^{5}$ \\ Sung Whan $\mathrm{Ha}, \mathrm{MD}^{1}$
}

\section{Purpose}

This study evaluated the effect of surgery-radiotherapy interval (SRI) on outcomes in patients treated with adjuvant radiotherapy (RT) after breast-conserving surgery (BCS) and adjuvant four cycles of doxorubicin/cyclophosphamide (AC) followed by four cycles of taxane.

\section{Materials and Methods}

From 1999 to 2007, 397 eligible patients were diagnosed. The effect of SRI on outcomes was analyzed using a Cox proportional hazards model, and a maximal chi-square method was used to identify optimal cut-off value of SRI for each outcome.

\section{Results}

The median SRI was 6.7 months (range, 5.6 to 10.3 months). A SRI of 7 months was the significant cut-off value for distant metastasis-free survival (DMFS) and disease-free survival (DFS) using a maximal chi-square method. For overall survival, a significant cut-off value was not found. The patients with SRI $>7$ months had worse 6-year DMFS and DFS than those with $\mathrm{SRI} \leq 7$ months on univariate analysis (DMFS, $81 \%$ vs. $91 \%, \mathrm{p}=0.003$; DFS, $78 \%$ vs. $89 \%, p=0.002)$. On multivariate analysis, SRI $>7$ months did not affect DMFS and DFS.

\section{Conclusion}

RT delayed for more than 7 months after BCS and adjuvant four cycles of AC followed by four cycles of taxane did not compromise clinical outcomes.

\author{
${ }^{1}$ Department of Radiation Oncology, \\ Seoul National University \\ College of Medicine, Seoul, \\ ${ }^{2}$ Department of Radiation Oncology, \\ Konkuk University Medical Center, Seoul, \\ ${ }^{3}$ Center for Breast Cancer, \\ Research Institute and Hospital, \\ National Cancer Center, Goyang, \\ Departments of ${ }^{4}$ Surgery and \\ ${ }^{5}$ Internal Medicine, \\ Seoul National University \\ College of Medicine, Seoul, Korea
}

\section{Key words}

Breast neoplasms, Segmental mastectomy, Time-to-treatment, Radiotherapy, Adjuvant chemotherapy

\author{
Correspondence: Kyung Hwan Shin, MD \\ Department of Radiation Oncology, \\ Seoul National University College of Medicine, \\ 101 Daehak-ro, Jongno-gu, Seoul 03080, Korea \\ Tel: $82-2-2072-2524$ \\ Fax: 82-2-765-3317 \\ E-mail: radiat@snu.ac.kr \\ Received March 19, 2015 \\ Accepted April 22, 2015 \\ Published Online June 5, 2015
}




\section{Introduction}

Since the Cancer and Leukemia Group B (CALGB) 9344 and the National Surgical Adjuvant Breast and Bowel Project (NSABP) B28 trials were reported, four cycles of doxorubicin/cyclophosphamide (AC) followed by four cycles of taxane has become a standard adjuvant chemotherapy regimen for patients with node-positive breast cancer [1,2]. The addition of taxane to AC improved disease-free survival (DFS), overall survival (OS), and local control (LC) despite a prolonged surgery-radiotherapy interval (SRI) [3] .

However, delays between treatments can be harmful due to tumor doubling time, and delayed SRI can increase the development of radioresistance $[4,5]$. Several studies have identified a relationship between SRI and treatment outcomes in breast cancer. Most studies analyzed a heterogeneous population in terms of disease stage, nodal status, type of surgery, adjuvant chemotherapy, and adjuvant endocrine therapy. Hence, the results of these studies are contradictory, and the maximal safe SRI has not been established.

The aim of the present study is to examine the effect of SRI on treatment outcomes in breast cancer patients treated with breast-conserving surgery (BCS) and adjuvant chemotherapy with four cycles of AC plus four cycles of taxane.

\section{Materials and Methods}

\section{Patient population}

From October 1999 to June 2007, 397 patients with nodepositive breast cancer underwent BCS, adjuvant four cycles of AC followed by four cycles of taxane, and adjuvant radiotherapy (RT) in sequence at the National Cancer Center and Seoul National University Hospital, Korea.

\section{Treatment}

All patients had BCS with axillary lymph node dissection or sentinel lymph node biopsy. The original pathologic report was reviewed to collect histologic data. All patients received four cycles of doxorubicin $60 \mathrm{mg} / \mathrm{m}^{2}$ by slow intravenous push and then cyclophosphamide $600 \mathrm{mg} / \mathrm{m}^{2}$ intravenous infusion over 30 minutes every 21 days followed by four additional 21-day cycles of taxane (paclitaxel $175 \mathrm{mg} / \mathrm{m}^{2}$ intravenous or docetaxel $75 \mathrm{mg} / \mathrm{m}^{2}$ intravenous as a 3-hour infusion on day 1 of each cycle).

Adjuvant RT was delivered after chemotherapy was completed. All patients underwent whole breast RT using
6-15 MV photons. Seven patients were treated with hypofractionated RT of 39 Gy using 3 Gy per fraction and the rest of the patients received conventional RT of 50-50.4 Gy at 1.8-2.0 Gy per fraction. An additional 9-16.2 Gy electron-boost using 1.8-3.0 Gy/fraction was delivered to the tumor bed. The addition of regional RT was decided at the discretion of the treating radiation oncologist's assessment of regional failure risk. Two hundred and seventeen patients $(54.7 \%)$ received 45-50.4 Gy at 1.8-2.0 Gy/fraction to the axillary and supraclavicular (SCL) regions. Among these patients, only one patient received additional RT to the internal mammary node (IMN) region.

Adjuvant hormonal therapy (HT) was given to most patients with estrogen receptor (ER)-positive or progesterone receptor (PR)-positive tumors. Three hundred and eight patients (77.6\%) had ER-positive or PR-positive tumors. Among these patients, 251 received tamoxifen, 46 received aromatase inhibitors, 10 did not receive $\mathrm{HT}$, and one patient had unavailable data. One patient received tamoxifen without hormonal receptor expression. If adjuvant HT was indicated, it was given with RT in most cases.

There were 71 patients (17.9\%) with HER2-positive (immunohistochemistry $3+$ or fluorescence in situ hybridization positive) tumors. Fifteen of these patients were treated with adjuvant trastuzumab and four patients received adjuvant lapatinib. Adjuvant trastuzumab or lapatinib were given on a 21-day cycle for one year and could be used with RT.

\section{Statistical analysis}

The interval between surgery and RT was defined as the time from the date of the first oncologic surgery to the first day of RT. The definition of ipsilateral breast tumor recurrence (IBTR) was recurrence of invasive breast cancer or ductal carcinoma in situ in the same breast treated with RT. Loco-regional recurrence (LRR) included IBTR, ipsilateral axillary lymph node recurrence, IMN recurrence, and SCL recurrence. Failure at any other site was considered a distant metastasis (DM). Each failure was scored as an event regardless of recurrence at other sites. Contralateral breast tumor recurrence was not counted as a failure but as a secondary malignancy. An event for DFS included IBTR, LRR and DM. The Kaplan-Meier method was used to calculate the actuarial rates of ipsilateral breast tumor recurrence-free survival (IBTRFS), loco-regional recurrence-free survival (LRRFS), distant metastasis-free survival (DMFS), DFS, and OS. Comparison between groups was performed by two-sided log-rank tests. Cox proportional hazards model was used for multivariate analysis and analysis of the effect of SRI on clinical outcomes. Maxstat, a maximal chi-square method in $\mathrm{R}$ 2.13.0 (R Development Core Team, Vienna, Austria, http:// www.R-project.org) was used to identify optimal cut-off 
Table 1. Patient and tumor characteristics

\begin{tabular}{|c|c|}
\hline Variable & No. of patients $(\%)$ \\
\hline \multicolumn{2}{|l|}{ Age (yr) } \\
\hline$<40$ & $87(21.9)$ \\
\hline$\geq 40$ & $310(78.1)$ \\
\hline \multicolumn{2}{|l|}{ Histology } \\
\hline IDCa & $375(94.5)$ \\
\hline ILCa & $6(1.5)$ \\
\hline Others & $16(4.0)$ \\
\hline \multicolumn{2}{|l|}{ Tumor location } \\
\hline Right breast & $183(46.1)$ \\
\hline Left breast & $214(53.9)$ \\
\hline \multicolumn{2}{|l|}{ Tumor size (cm) } \\
\hline$\leq 2$ & $166(41.8)$ \\
\hline$>2$ & $231(58.2)$ \\
\hline \multicolumn{2}{|l|}{ Tumor grade ${ }^{a)}$} \\
\hline I & $13(3.3)$ \\
\hline II & $188(47.8)$ \\
\hline III & $192(48.9)$ \\
\hline \multicolumn{2}{|l|}{ Resection margin } \\
\hline Negative & $343(86.4)$ \\
\hline Close $(\leq 2 \mathrm{~mm})$ & 48 (12.1) \\
\hline Positive & $6(1.5)$ \\
\hline \multicolumn{2}{|l|}{ EIC $^{\text {a) }}$} \\
\hline No & $247(68.4)$ \\
\hline Yes & $114(31.6)$ \\
\hline \multicolumn{2}{|l|}{ LVI $^{a)}$} \\
\hline No & $53(14.4)$ \\
\hline Yes & $314(79.1)$ \\
\hline \multicolumn{2}{|l|}{ Ki-67 (\%) } \\
\hline$\leq 15$ & $244(64.0)$ \\
\hline$>15$ & $137(36.0)$ \\
\hline \multicolumn{2}{|l|}{ Estrogen receptor } \\
\hline Negative & $114(28.7)$ \\
\hline Positive & $283(71.3)$ \\
\hline \multicolumn{2}{|l|}{ Progesterone receptor } \\
\hline Negative & $122(30.7)$ \\
\hline Positive & $275(69.3)$ \\
\hline \multicolumn{2}{|l|}{ HER-2 receptora) } \\
\hline Negative & $325(82.1)$ \\
\hline Positive $^{\text {b) }}$ & $71(17.9)$ \\
\hline \multicolumn{2}{|l|}{ Menopause status $^{\text {a) }}$} \\
\hline Premenopausal & $228(59.4)$ \\
\hline Postmenopausal & $125(32.6)$ \\
\hline Perimenopausal & $31(8.1)$ \\
\hline \multicolumn{2}{|l|}{ Stage by AJCC 7th edition } \\
\hline IIA (T1N1) & $115(29.0)$ \\
\hline IIB (T2N1) & $131(33.0)$ \\
\hline IIIA (T1-2N2,T3N1-2) & $98(24.7)$ \\
\hline IIIB (T4N1-2) & $1(0.3)$ \\
\hline IIIC (N3) & $52(13.0)$ \\
\hline
\end{tabular}

Table 1. Continued

\begin{tabular}{lc} 
Variable & No. of patients (\%) \\
\hline Nodal stage (pathological) & \\
N1mi & $13(3.3)$ \\
N1 & $237(59.7)$ \\
N2 & $95(23.9)$ \\
N3 & $52(13.1)$ \\
Radiation field & \\
Breast only & $180(45.3)$ \\
Breast and regional lymphatics & $217(54.7)$ \\
\hline
\end{tabular}

IDCa, invasive ductal carcinoma; ILCa, invasive lobular carcinoma; EIC, extensive intraductal component; LVI, lymphovascular space invasion; AJCC, American Joint

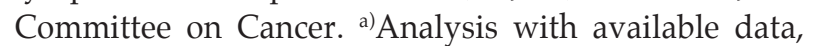
b)Immunohistochemistry 3+ or fluorescence in situ hybridization positive.

value of SRI for each outcome. Two-tailed null hypotheses of no difference were rejected if $p$-values were less than 0.05 , or, equivalently, if the $95 \%$ confidence intervals (CIs) of hazard ratio estimates excluded 1 . All statistical analyses, except Maxstat, were carried out using SPSS ver. 16.0 (SPSS Inc., Chicago, IL).

\section{Results}

\section{Patient characteristics}

Table 1 summarizes the patient and tumor characteristics. The median age of patients at diagnosis was 45 years old (range, 22 to 73 years). Most patients had invasive ductal carcinoma $(94.5 \%)$ according to the World Health Organization's classification system. Positive or close resection margin was reported in $13.6 \%$ of all cases following the last surgery. There were 192 patients (48.9\%) with histologic grade III tumor, 114 patients $(31.6 \%)$ with extensive intraductal component (EIC), 314 patients $(79.1 \%)$ with lymphovascular space invasion (LVI) and 137 patients $(36.0 \%)$ with Ki-67 greater than 15\%. The pathologic stage by American Joint Committee on Cancer (AJCC) seventh edition was IIA in 115 patients $(29.0 \%)$, IIB in 131 patients $(33.0 \%)$, IIIA in 98 patients $(24.7 \%)$, IIIB in one patient $(0.3 \%)$, and IIIC in 52 patients (13.0\%). 


\section{Outcomes}

The median follow-up duration of all patients was 65 months (range, 9 to 137 months). During follow-up, there were 12 cases of IBTR, 20 cases of LRR, 45 cases of DM, and 24 deaths. The actuarial 6-year IBTRFS was 96.9\%, LRRFS 93.8\%, DMFS $87.6 \%$, DFS $85.5 \%$, and OS $93.9 \%$.

\section{Surgery-radiotherapy interval}

The median interval between surgery and RT was 6.7 months (range, 5.6 to 10.3 months). Because the association between SRI as a continuous variable and clinical outcomes should be confirmed before finding an optimal cut-off value using maximal chi-square method, a Cox proportional hazard model was performed to estimate the association of SRI with each clinical outcome (Table 2). An increase of SRI was related to worse DMFS ( $\mathrm{p}=0.002)$, DFS $(\mathrm{p}=0.003)$, and OS $(\mathrm{p}=0.008)$. The optimal cut-off value of SRI for DMFS and DFS was 7 months by maximal chi-square method ( $\mathrm{p}=0.024$ and $\mathrm{p}=0.016$, respectively) (Figs. 1 and 2). Even though the optimal cut-off value of SRI for OS was 7.5 months, it was not statistically significant $(\mathrm{p}=0.255)$. When a SRI of 7 months was applied, there were 275 patients in the group with a SRI $\leq 7$ months and 122 patients in the group with SRI $>7$ months. The 6-year actuarial DMFS for patients with SRI $>7$ months was $81 \%$ versus $91 \%$ for patients with $\mathrm{SRI} \leq 7$ months $(\mathrm{p}=0.003)$, and DFS was $78 \%$ versus $89 \%(\mathrm{p}=0.002)$, respectively (Table 3).

\section{Univariate analysis and multivariate analysis}

Table 3 provides univariate and multivariate analyses of prognostic factors for 6-year outcomes. Patients younger than 40 years old, ER-negative, and PR-negative tumors showed poor prognosis in IBTRFS and LRRFS. The 6-year IBTRFS for patients age $<40$ years versus $\geq 40$ years was $93 \%$ versus $98 \%(\mathrm{p}=0.001)$ and the 6-year LRRFS was $91 \%$ versus 95\% ( $\mathrm{p}=0.037)$, respectively. The 6-year IBTRFS for patients with ER-negative tumors versus ER-positive tumors was 93\% versus $99 \%(\mathrm{p}=0.001)$ and the 6 -year LRRFS was $87 \%$ versus $97 \%$ ( $\mathrm{p}<0.001)$, respectively. PR-negative tumors showed worse 6-year IBTRFS than PR-positive tumors (93\% for PR-negative vs. 99\% for PR-positive, $\mathrm{p}<0.001$ ) and worse 6-year LRRFS (89\% for PR-negative vs. 97\% for PR-positive, $\mathrm{p}<0.001$ ). Histology, tumor size, tumor grade, resection margin, EIC, LVI, HER-2, menopause status, Ki-67, nodal stage and stage group did not have an impact on IBTRFS and LRRFS.

Tumor size, tumor grade, ER status, PR status, nodal stage, and stage group were analyzed as prognosticators for the 6-year DMFS and DFS. Larger tumors were a poor prognostic factor for DMFS (95\% for tumor size $\leq 2 \mathrm{~cm}$ vs. $82 \%$ for tumor size $>2 \mathrm{~cm}, \mathrm{p}<0.001)$ and for DFS $(93 \%$ for tumor size $\leq 2 \mathrm{~cm}$ vs. $81 \%$ for tumor size $>2 \mathrm{~cm}, \mathrm{p}=0.001$ ). Higher tumor grade negatively affected DMFS (100\% for grade I vs. $92 \%$ for grade II vs. $82 \%$ for grade III, $\mathrm{p}=0.003)$ and DFS (100\% for grade I vs. $91 \%$ for grade II vs. $79 \%$ for grade III, $\mathrm{p}=0.006$ ). ER-negative tumors showed worse 6-year DMFS than ERpositive tumors (79\% for ER-negative vs. 91\% for ER-positive, $\mathrm{p}<0.001)$ and 6-year DFS (75\% for ER-negative vs. $90 \%$ for ER-positive, $p<0.001)$. The 6-year DMFS for patients with PR-negative tumors versus PR-positive tumors was $83 \%$ versus $90 \%(\mathrm{p}=0.029)$ and the 6 -year DFS was $78 \%$ versus $89 \%$ ( $\mathrm{p}=0.001)$, respectively. Higher nodal stage was a negative prognosticator for DMFS ( $94 \%$ for N1 vs. $80 \%$ for N2 vs. $72 \%$ for N3, p < 0.001) and DFS ( $91 \%$ for N1 vs. $77 \%$ for N2 vs. $72 \%$ for N3, p < 0.001). Higher stage group presented poorer prognosis for DMFS ( $94 \%$ for stage II vs. $77 \%$ for stage III, $\mathrm{p}<0.001$ ) and DFS (99\% for stage II vs. $86 \%$ for stage III, $\mathrm{p}<0.001$ ).

Larger tumor ( $97 \%$ for tumor size $\leq 2 \mathrm{~cm}$ vs. $91 \%$ for tumor size $>2 \mathrm{~cm}, \mathrm{p}=0.012$ ), ER-negative tumor ( $96 \%$ for ER-positive vs. $88 \%$ for ER-negative, $\mathrm{p}<0.001$ ), higher nodal stage ( $99 \%$ for $\mathrm{N} 1$ vs. $87 \%$ for $\mathrm{N} 2$ vs. $85 \%$ for N3, p < 0.001), and higher stage group (99\% for stage II vs. $86 \%$ for stage III, $\mathrm{p}<0.001$ ) negatively affected OS.

A multivariate analysis was performed to exclude con-

Table 2. The effect of SRI on clinical outcomes and optimal cut-off value of SRI

\begin{tabular}{lccc} 
& p-value of cox proportional hazards model & Optimal cut-off value (mo) & p-value of maximal chi-square test \\
\hline IBTRFS & 0.239 & - & - \\
LRRFS & 0.110 & - & - \\
DMFS & 0.002 & 7 & 0.024 \\
DFS & 0.003 & 7 & 0.016 \\
OS & 0.008 & 7.5 & 0.255 \\
\hline
\end{tabular}

SRI, surgery-radiotherapy interval; IBTRFS, ipsilateral breast tumor recurrence-free survival; LRRFS, locoregional recurrence-free survival; DMFS, distant metastasis-free survival; DFS, disease-free survival; OS, overall survival. 


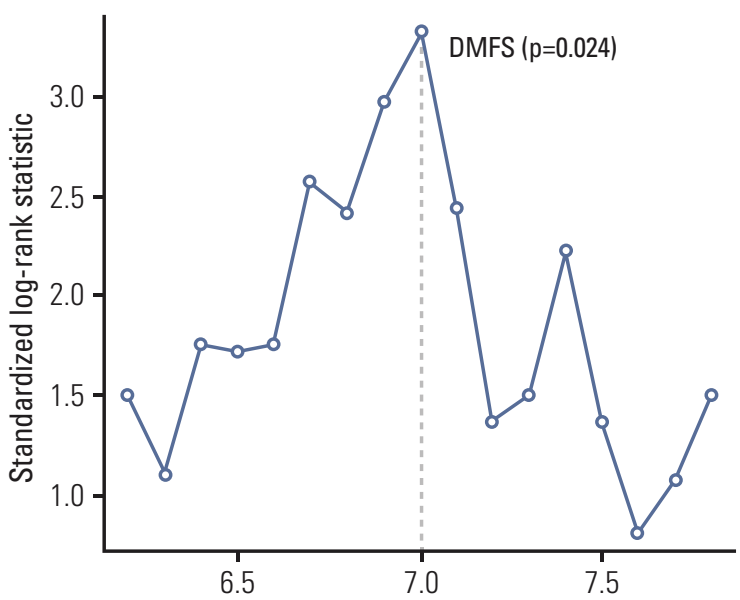

$\mathrm{SRI}(\mathrm{mo})$

Fig. 1. Maximal chi-square method for distant metastasisfree survival (DMFS) $(p=0.024)$. The cut-off value of surgery-radiotherapy interval (SRI) was set to what provided the best separation of DMFS into two groups, where the standardized log-rank statistics take their maximum.

founding factors for outcomes. In multivariate analysis, patients younger than 40 years old had a worse prognosis for IBTRFS; tumor size more than $2 \mathrm{~cm}$ had worse prognosis for DMFS; nodal stage was an independent prognostic factor for OS. A SRI of greater than 7 months, however, lost its prognostic significance on multivariate analysis.

\section{Discussion}

In the present study, the actuarial 6-year IBTRFS, LRRFS, DMFS, DFS, and OS rates for patients treated with BCS, adjuvant $\mathrm{AC}$ plus taxane, and adjuvant RT in sequence were $96.9 \%, 93.8 \%, 87.6 \%, 85.5 \%$, and $93.9 \%$, respectively. RT delayed for more than 7 months did not affect clinical outcomes in this population.

Since the introduction of adjuvant chemotherapy in breast cancer, many clinicians have studied the optimal SRI for patients treated with adjuvant chemotherapy. In 1993, Buchholz et al. [6] arbitrarily divided patients who received various types of chemotherapy regimens into two groups: SRI $\leq 6$ months and $>6$ months. They showed that SRI $>$ 6 months negatively affected LC, DFS, and OS. Review articles have been published on the optimal timing of adjuvant $\mathrm{RT}$ in breast cancer [7-10]. These researchers concluded that

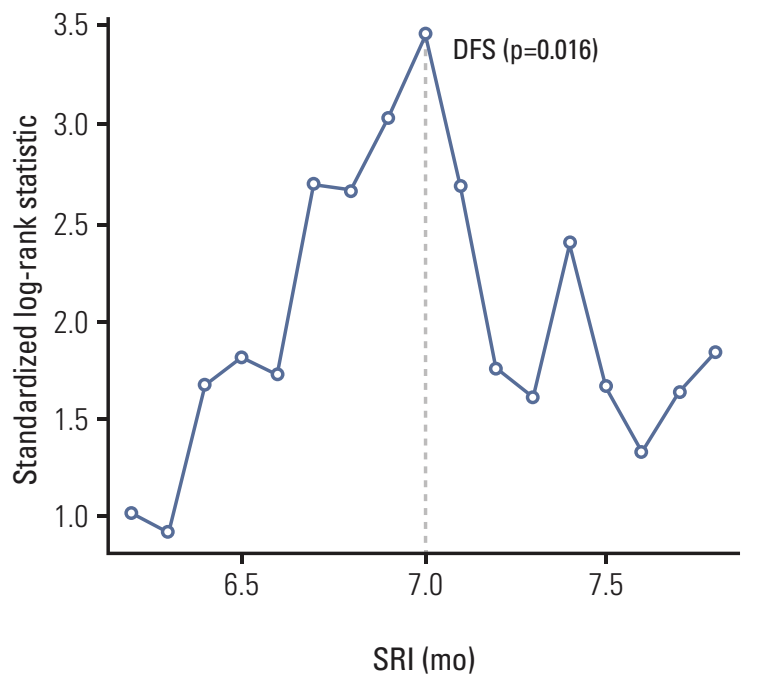

Fig. 2. Maximal chi-square method for disease-free survival (DFS) ( $p=0.016)$. The cut-off value of surgery-radiotherapy interval (SRI) was set to what provided the best separation of DFS into two groups, where the standardized log-rank statistics take their maximum.

SRI should not exceed 20-24 weeks after surgery when adjuvant chemotherapy was given [7-9]. A recent Cochrane review concluded that breast cancer outcomes were not compromised regardless of the sequence of adjuvant treatments within 7 months after surgery [10]. Among the patients treated with adjuvant chemotherapy in these review articles, most received cyclophosphamide, methotrexate, and 5-fluorouracil (CMF) regimen.

Although there is some evidence on SRI for patients who received adjuvant chemotherapy as mentioned above, there are no analyses of SRI in patients treated exclusively with four cycles of AC plus four cycles of taxane. In general, the $\mathrm{AC}$ plus taxane regimen takes longer to complete than a CMF regimen. However, CALGB 9344 demonstrated that the addition of a taxane to AC for node-positive breast cancer improved LC as well as DFS and OS [3]. Therefore, an acceptable SRI for AC plus taxane regimen needs to be established.

There have been studies of the relationship between SRI and LC for breast cancer patients treated with adjuvant chemotherapy regimens including anthracycline. Livi et al. [11] performed a subgroup analysis on patients treated with adjuvant chemotherapy ( $65 \%$ with 6 cycles of CMF regimen, $20 \%$ with anthracycline-based regimen and $15 \%$ with other regimens), adjuvant RT and no HT. Multivariate analysis showed RT timing was an independent prognostic factor for local relapse especially in the group of SRI > 180 days 


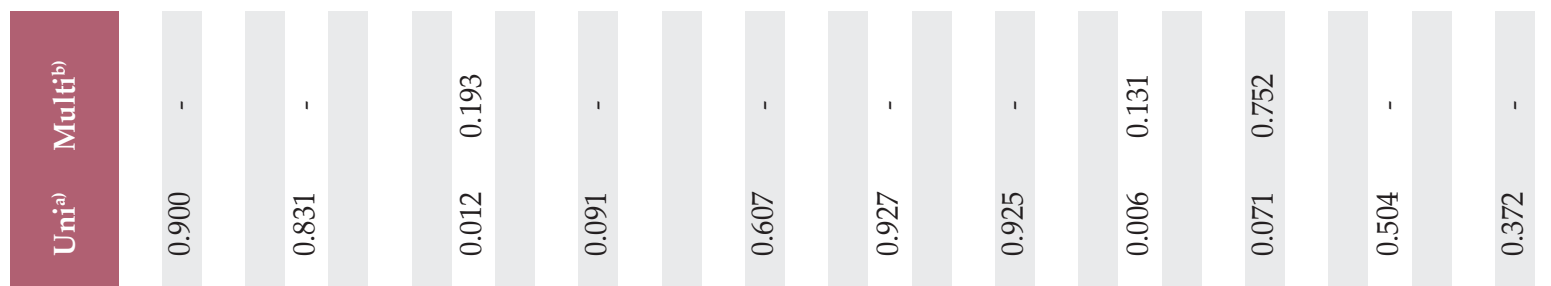

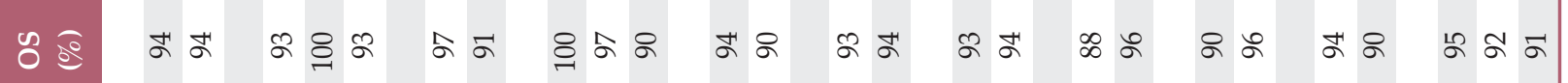

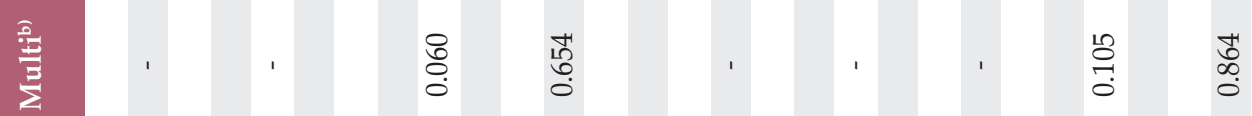

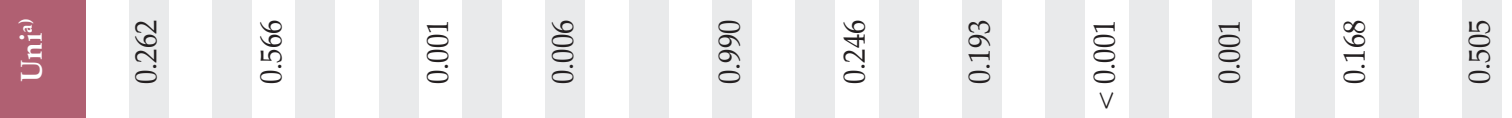

崩

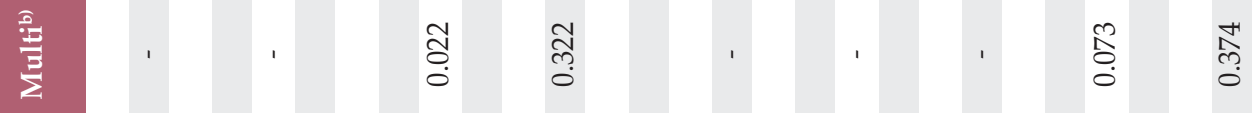

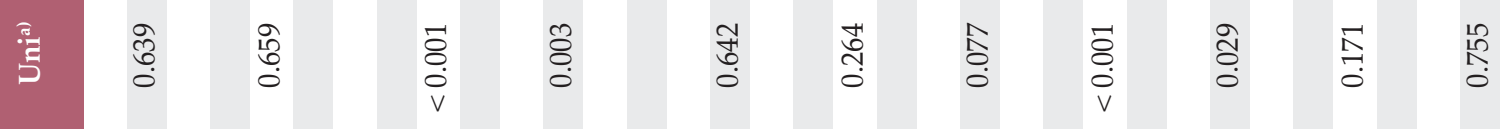

車

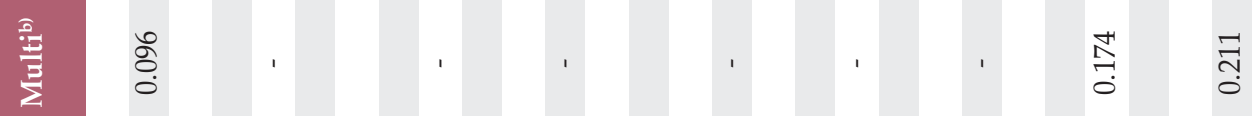

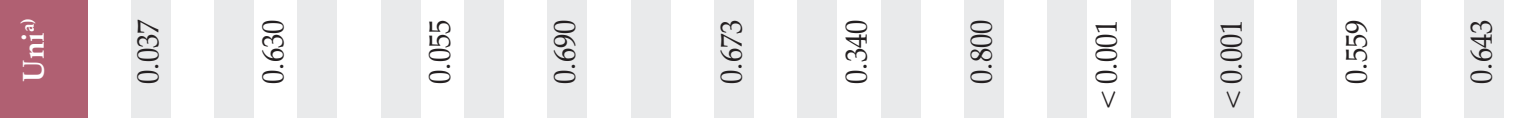

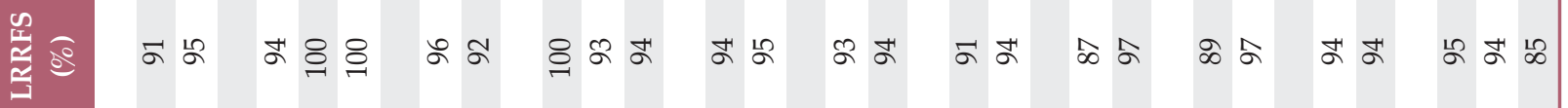

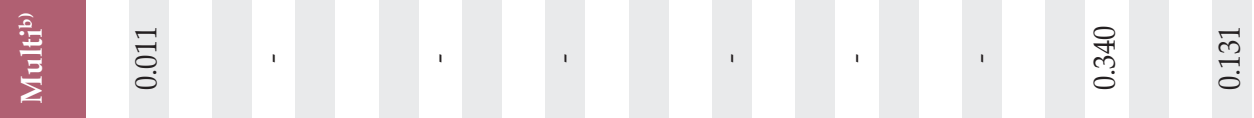

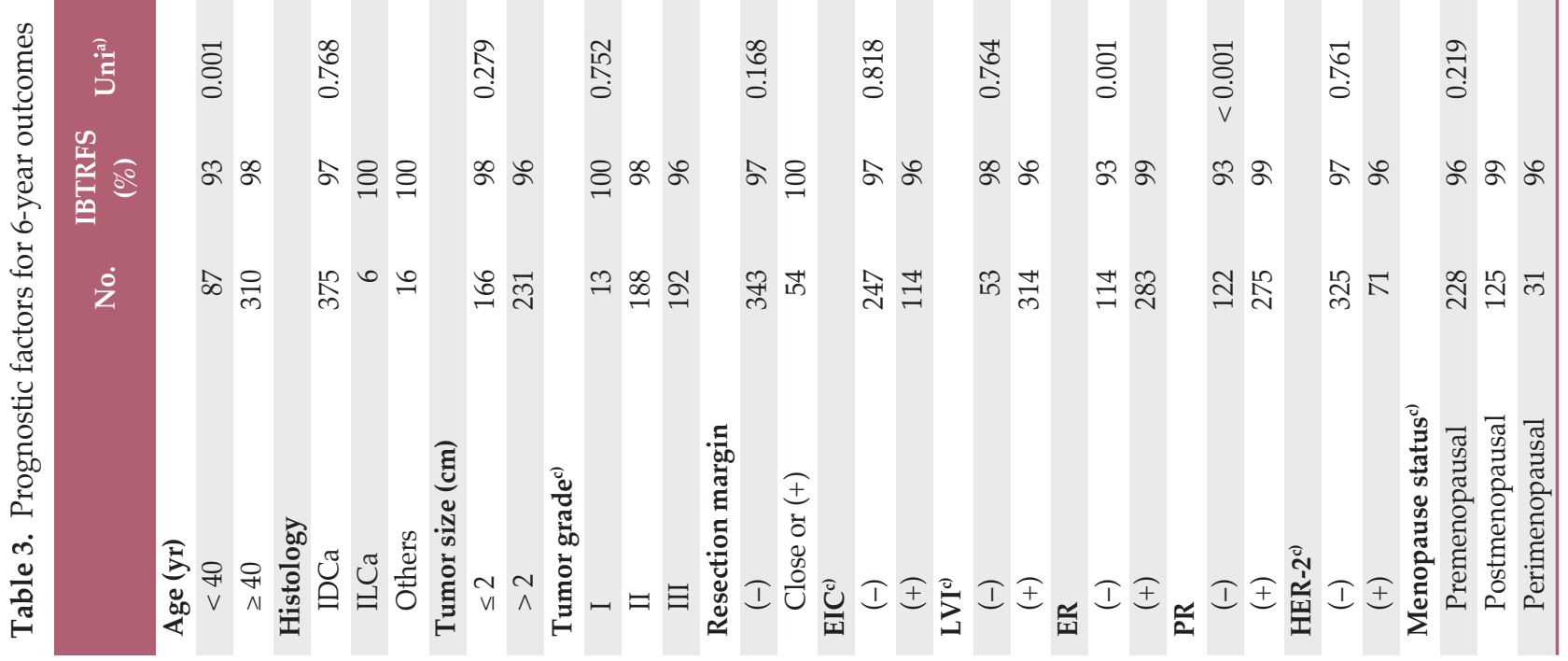




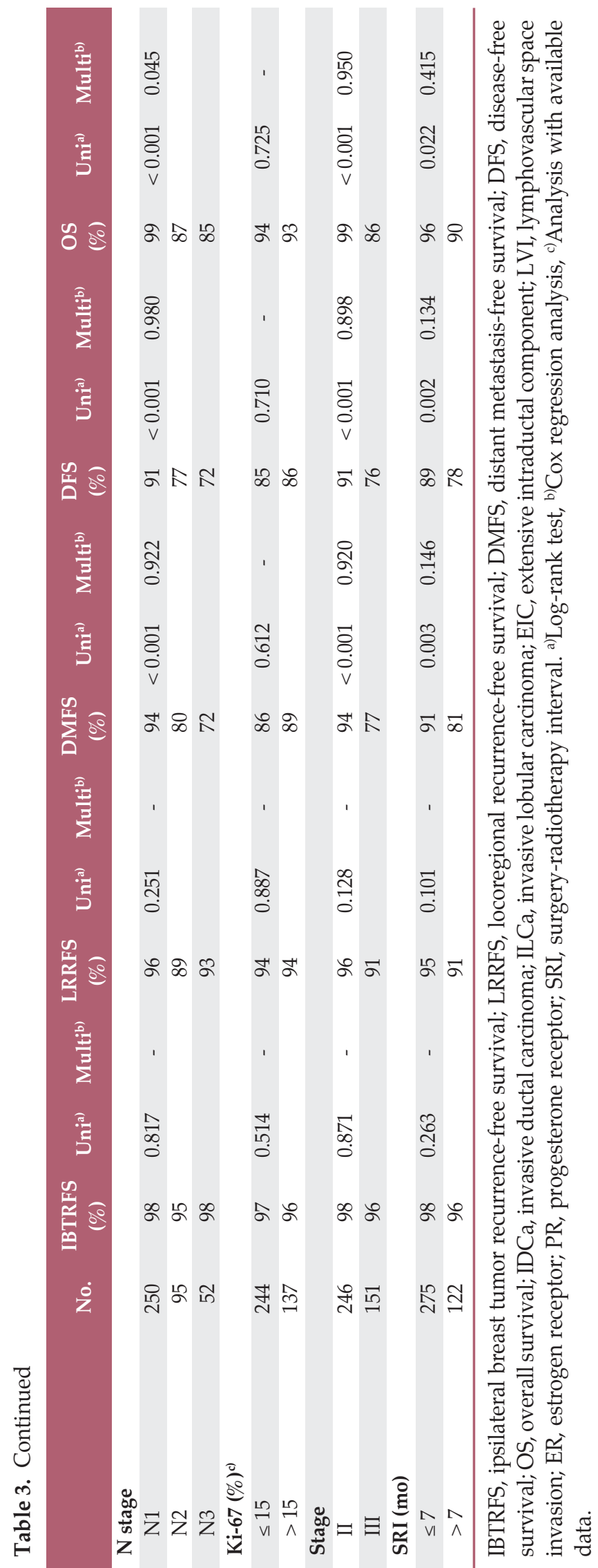

$(\mathrm{p}=0.045)$. However, other studies have shown that SRI was not associated with LC. Yock et al. [12] suggested that more than 7 months of SRI did not compromise LC in patients who received four or six cycles of CMF or AC regimen. Cefaro et al. [13] also concluded that SRI $\geq 6$ months did not affect LC in patients who underwent an unspecified number of cycles of CMF or an anthracycline-based regimen. Corradini et al. [14] showed delayed RT did not compromise LRRFS in the chemotherapy group in a cohort study of 1,393 patients. In the present study, we found that SRI was not associated with IBTRFS and LRRFS in Cox proportional hazards model for node-positive breast cancer patients treated with BCS and four cycles of AC followed by four cycles of taxane.

As for other outcomes, there was no association between SRI and DMFS, DFS, and / or OS. Two studies indicated that SRI was not associated with DFS and OS in breast cancer patients treated with adjuvant chemotherapy before RT $[5,12]$. Yock et al. [12] showed that SRI had no effect on distant failure. Corradini et al. [14] suggested SRI $\geq 24$ weeks did not compromise OS. Downing et al. [15] used path analysis to investigate the relationship among survival, SRI and receipt of adjuvant chemotherapy before RT or after RT. They also concluded that SRI was not related to survival in the path model (hazard ratio, 1.00; 95\% CI, 0.99 to 1.01 per week increase). In the current study, the optimal cut-off value of SRI for DMFS and DFS was 7 months, and there was no significant optimal cut-off value of SRI for OS. More than 7 months of SRI resulted in poor prognosis for DMFS and DFS on univariate analysis, but the significance was lost on multivariate analysis. Therefore, 7 months of SRI seemed to have no significant effect on clinical outcomes in our study population.

Our study has both limitations and strengths. Given the retrospective nature of the study, the potential for selection bias cannot be excluded. Our study has a smaller sample size and shorter follow up period than previous studies of SRI or outcomes in breast cancer [5,11,14]. However, the study population is homogeneous in respect to the treatment.

\section{Conclusion}

More than 7 months of SRI did not compromise clinical outcomes of node-positive breast cancer patients treated with $\mathrm{BCS}$, adjuvant $\mathrm{AC}$ followed by taxane, and RT in sequence. Because it is clearly unethical to randomize SRI among patients who receive the same treatment regimen, future research is needed on a larger data set to confirm the effect of SRI on the treatment outcomes in breast cancer. 


\section{Conflicts of Interest}

Conflict of interest relevant to this article was not reported.

\section{References}

1. Henderson IC, Berry DA, Demetri GD, Cirrincione CT, Goldstein LJ, Martino S, et al. Improved outcomes from adding sequential paclitaxel but not from escalating doxorubicin dose in an adjuvant chemotherapy regimen for patients with nodepositive primary breast cancer. J Clin Oncol. 2003;21:976-83.

2. Mamounas EP, Bryant J, Lembersky B, Fehrenbacher L, Sedlacek SM, Fisher B, et al. Paclitaxel after doxorubicin plus cyclophosphamide as adjuvant chemotherapy for node-positive breast cancer: results from NSABP B-28. J Clin Oncol. 2005;23:3686-96.

3. Sartor CI, Peterson BL, Woolf S, Fitzgerald TJ, Laurie F, Turrisi AJ, et al. Effect of addition of adjuvant paclitaxel on radiotherapy delivery and locoregional control of node-positive breast cancer: cancer and leukemia group B 9344. J Clin Oncol. 2005; 23:30-40.

4. Johnson A. The timing of treatment in breast cancer: gaps and delays in treatment can be harmful. Breast Cancer Res Treat. 2000;60:201-9.

5. Karlsson P, Cole BF, Colleoni M, Roncadin M, Chua BH, Murray $\mathrm{E}$, et al. Timing of radiotherapy and outcome in patients receiving adjuvant endocrine therapy. Int J Radiat Oncol Biol Phys. 2011;80:398-402.

6. Buchholz TA, Austin-Seymour MM, Moe RE, Ellis GK, Livingston RB, Pelton JG, et al. Effect of delay in radiation in the combined modality treatment of breast cancer. Int J Radiat Oncol Biol Phys. 1993;26:23-35.

7. Tsoutsou PG, Koukourakis MI, Azria D, Belkacemi Y. Optimal timing for adjuvant radiation therapy in breast cancer: a comprehensive review and perspectives. Crit Rev Oncol Hematol. 2009;71:102-16.
8. Ruo Redda MG, Verna R, Guarneri A, Sannazzari GL. Timing of radiotherapy in breast cancer conserving treatment. Cancer Treat Rev. 2002;28:5-10.

9. Huang J, Barbera L, Brouwers M, Browman G, Mackillop WJ. Does delay in starting treatment affect the outcomes of radiotherapy? A systematic review. J Clin Oncol. 2003;21:555-63.

10. Hickey BE, Francis DP, Lehman M. Sequencing of chemotherapy and radiotherapy for early breast cancer. Cochrane Database Syst Rev. 2013;4:CD005212.

11. Livi L, Borghesi S, Saieva C, Meattini I, Rampini A, Petrucci A, et al. Radiotherapy timing in 4,820 patients with breast cancer: university of florence experience. Int J Radiat Oncol Biol Phys. 2009;73:365-9.

12. Yock TI, Taghian AG, Kachnic LA, Coen JJ, Assaad SI, Powell $\mathrm{SN}$. The effect of delaying radiation therapy for systemic chemotherapy on local-regional control in breast cancer. Breast Cancer Res Treat. 2004;84:161-71.

13. Cefaro GA, Genovesi D, Marchese R, Di Tommaso M, Di Febo $\mathrm{F}$, Ballone $\mathrm{E}$, et al. The effect of delaying adjuvant radiation treatment after conservative surgery for early breast cancer. Breast J. 2007;13:575-80.

14. Corradini S, Niemoeller OM, Niyazi M, Manapov F, Haerting $\mathrm{M}$, Harbeck N, et al. Timing of radiotherapy following breastconserving surgery: outcome of 1393 patients at a single institution. Strahlenther Onkol. 2014;190:352-7.

15. Downing A, Gilthorpe MS, Dodwell D, Lawrence G, Forman D. Waiting times for radiotherapy after breast-conserving surgery and the association with survival: a path analysis. Clin Oncol (R Coll Radiol). 2011;23:442-8. 